Please do not remove this page

RMIT

UNIVERSITY

\title{
Locative systems using mobile phones
}

Weight, Jenny

https://researchrepository.rmit.edu.au/esploro/outputs/9921863466201341/filesAndLinks?institution=61RMIT_INST\&index=null

Weight, J. (2008). Locative systems using mobile phones. IEEE Multimedia, 15(3), 6-9.

https://doi.org/10.1109/MMUL.2008.59

Published Version: https://doi.org/10.1109/MMUL.2008.59

Repository homepage: https://researchrepository.rmit.edu.au

(c) 2008 IEEE. Personal use of this material is permitted. However, permission to reprint/republish this material for advertising or promotional purposes or for creating new collective works for resale or redistribution to servers or lists, or to reuse any copyrighted component of this work in other works must be obtained from the IEEE.

Downloaded On 2023/04/26 21:17:55 +1000 


\section{Locative Systems Using Mobile Phones}

Jenny Weight RMIT University

Editor's Note

In the age of mobile media, artists interested in storytelling and place have been exploring various technologies to enable their locative media work. Jenny Weight traces the aesthetic and technical background to these works and discusses recent RMIT projects, which work with a solar powered Bluetooth server to deliver their poetic psychogeographic stories.

-Norie Neumark

specific media to a local population. This media is ambient because it's inscribed on the landscape, encountered as a function of passing through a location. Many technologies can be used to distribute locative media, and many are neither new nor novel: a signpost is a type of locative technology, and the words "Melbourne 42" is the sign's ambient media. Paintings, flyers, and live performances are locative because they are available at a specific location. If the performance is recorded and distributed via the Internet or television, that version is no longer location-specific.

Innovations in ambient media distribution use the mobile phone, a device that is always with us, always on, and therefore almost tailormade for such purposes. This article considers some ways in which mobile devices are used for locative distribution and discusses some of the technical and practical challenges. I will give examples of locative media/art projects including the mobile media group's work at RMIT.

The way media is distributed impacts the type of media produced; a signpost must be terse rather than poetic, for example. Media objects distributed to mobile phones from specific locations typically have these characteristics:

- environmental responsiveness, both spatially and temporally;
- content that uses peer-to-peer communication; and

- ambiguous privacy-you receive it in the public street to your private mobile phone.

As a result, such media can be tactical; like the Internet, it offers a way for individuals to engage with the public world. However most options for mobile phones' locative potential derive from marketing. For example, companies deliver offers by text message to nearby mobile phones. Another futuristic example is the famous scene in Steven Spielberg's Minority Report (2002) in which identity software based on the citizen's iris allows personalized holographic marketing. However, our group is interested in applications that are artistic, tactical, and pedagogical rather than marketing-oriented.

A history of locative media would include graffiti. Indeed, many locative projects using the mobile phone have taken inspiration from graffiti cultures. The problem with traditional graffiti is that the wrong people see it. Temporary graffiti, such as Graffiti Research Lab's L.A.S.E.R. Tagging System (see http:// www.graffitiresearchlab.com) partially overcomes problems derived from permanently marking public space. However, although such work is temporary and contextual, it remains unsubtle, nonprivate, and impersonal, and practices a large-scale aesthetic that eschews the intimacy of phone-based media. Paul Golding envisages phone-based, location-specific social software that enables selection of a specific community. ${ }^{1}$ Systems such as Dodgeball (see http://www.dodgeball.com/home) could perhaps be extended to include media distribution to a nominated set of people as they enter a location.

With such ambitions in mind, tagging and text-messaging systems permit the distribu- 
tion of intimate and potentially more personalized locative media. Yellow Arrow by Counts Media (see http://yellowarrow.net) uses locative tagging. Users text a code, which they find displayed on a yellow arrow affixed to the location, and receive a location-specific message in reply (see Figure 1). The technological complexity for this project is in its automated implementation of a text-messaging system. Authors have to buy their arrows.

Another approach uses a barcode. Barcode tagging is dependent on affixing a small visual object to the location, such as an individualized barcode, which acts as a link to a Web page. Users can access Web-based data by photographing the barcode with their phone and using tagging software. Two implementations of tagging are Semacode (see http:// semacode.org and Figure 2, next page) and Hypertag (see http://www.hypertag.com).

Semacode is free for noncommercial users. However, to be widely adopted, various technical and economic issues must be addressed. Furthermore, issues might vary for different telecommunications environments. In Australia, issues include

I data transfer costs;

I lack of inexpensive, mainstream, and compatible software and hardware;

I complexity of transfer to and from the phone.

Our group considers Bluetooth systems the most technically and economically viable of the options currently available.

\section{Solar-powered Bluetooth server}

Bluetooth is a localized wireless data-transfer network with a range of 10 to 20 meters. A Bluetooth server offers two-way, peer-to-peer communication and decentralized data storage. It can operate independently of telecommunications and even power infrastructure. This independence from infrastructure is particularly exciting to us, because it means that traditional media gatekeeping can be replaced by participatory cultures. At RMIT we are currently building a portable, solar-powered Bluetooth server (see Figures 3 and 4). The project has software, hardware, engineering, and industrial design aspects.

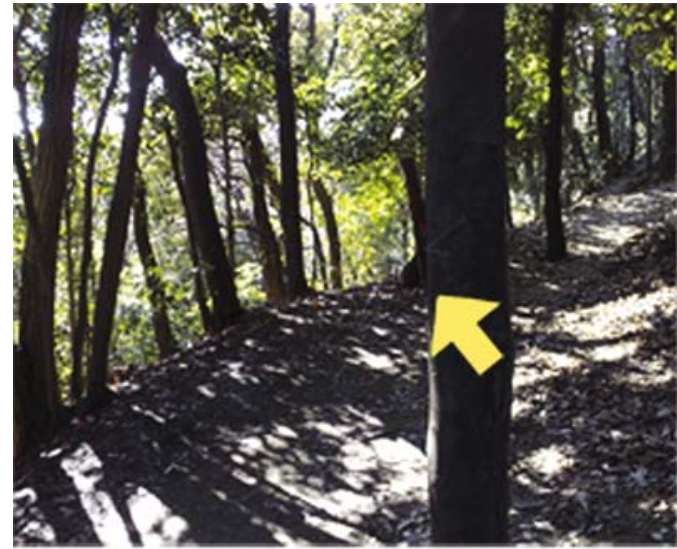

(a)
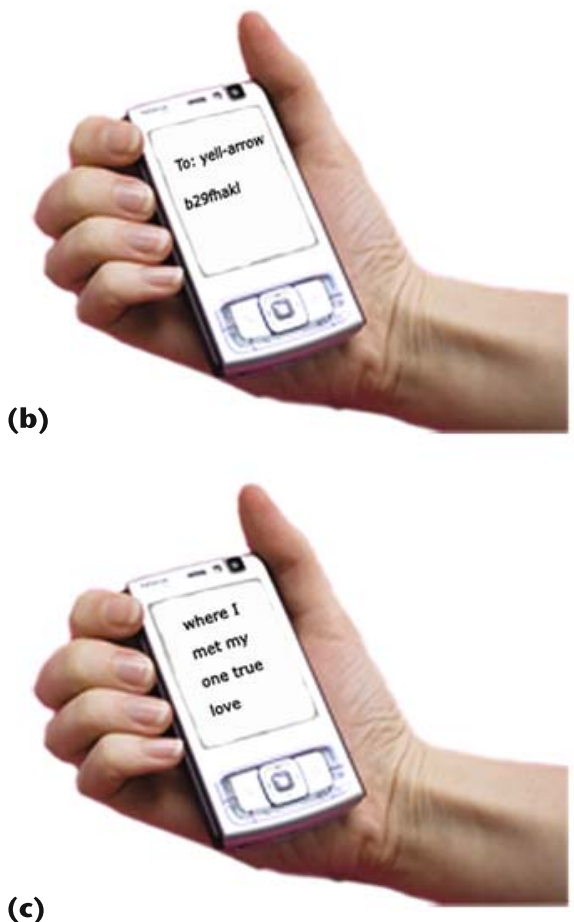

\section{Hardware}

We faced major challenges in ensuring that the server would withstand a great range of temperatures and at the same time, draw as little power as possible. Our choice was a 12volt mini PC. We chose the model because of its ability to withstand extreme temperatures and resist water splashes. Since making our choice, even more portable Bluetooth servers have come on the market; however, it's not clear that they have the programmable flexibility of the mini PC.

\section{Engineering}

Our tests have suggested the need for solar panels totaling 40 watts charging a 12 -volt battery at 18 amps per hour. However, at this
Figure 1.

Representation of the Yellow Arrow user experience: (a) the author attaches a yellow arrow with a code to a real-world object, (b) the viewer texts the code to a specified phone number, and (c) the viewer receives a text message about the location. 
Figure 2.

Representation of the Semacode user experience: (a) the viewer photographs the Semacode tag, generated at http:// semacode.com/tag; (b) the viewer accesses the URL that the tag points to; and (c) the Web page is loaded to the phone browser.

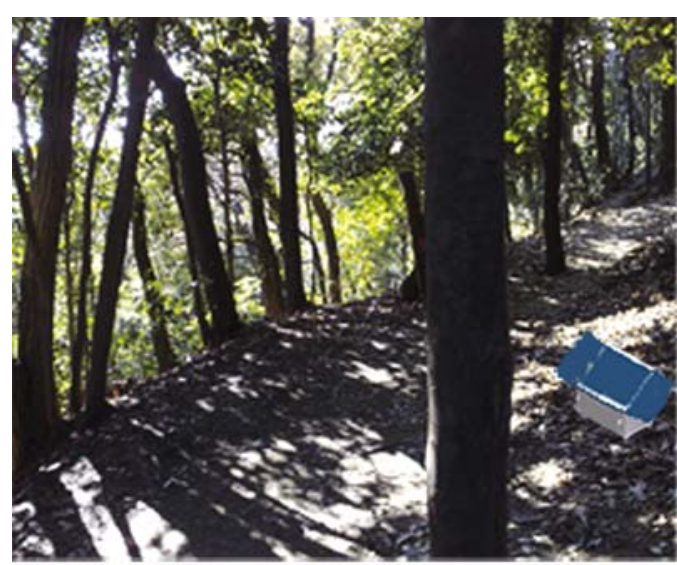

(a)

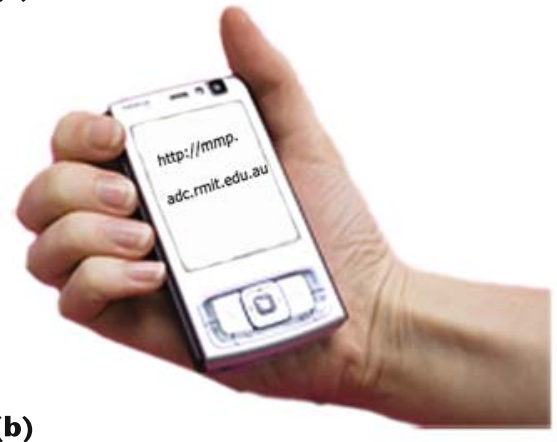

(b)

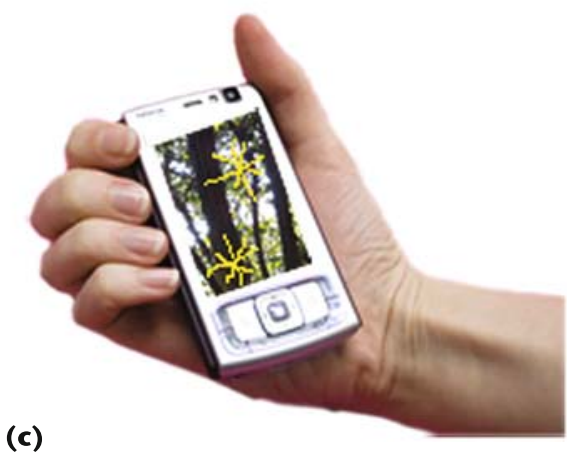

stage it's unlikely that the device will be completely independent of the public power grid.

The solar panels have a total area of approximately $1,000 \times 421 \times 23$ millimeters, a size that begins to impact the device's portability. Our solution is to have an angled lid, which has foldable wings when the device is transported (not yet installed). Sunlight should hit solar panels at a 90-degree angle. In the southern hemisphere, panels should face north. Given that the tilt of the panels is fixed, our compromise angle is the same as the latitude (in Melbourne, 38 degrees).

\section{Industrial design}

Another challenge is ventilation to cool the server, which at the same time must protect

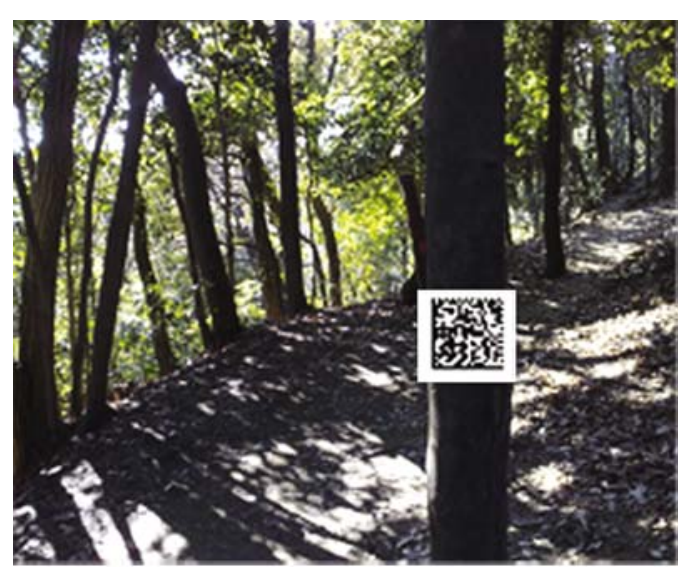

(a)

(b)
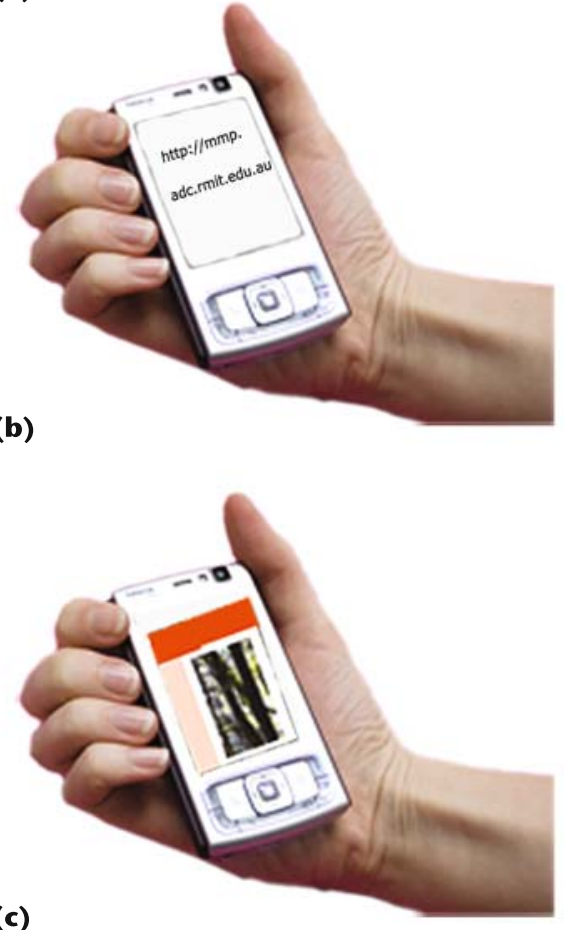

Figure 3. Representation of the Bluetooth server user experience: (a) server sends invitation to viewer's phone, (b) viewer accepts, and (c) media is sent to the phone.

the server from rain and splashing. Our solution entails a recessed floor.

\section{Software}

We used rapid prototyping as our project methodology. Our programmer, Ge PeiQi, started from the requirement analysis phase. The development environment is Windows Server 2003, Java 1.5, Eclipse 3.2, Bluecove framework, and Standard Widget Toolkit (SWT) framework. We used the Bluecove framework to implement the core Bluetooth 
component to communicate with the local and remote Bluetooth devices. We used the SWT framework to implement a GUI so the administrator could easily interact with the core component. Multithread was implemented to allow several requests to be processed concurrently.

By tracking what media is sent to what Bluetooth ID, we can ensure that the same phone will not receive the same media twice. In the future, we wish to permit users to receive an appropriate series of media items, all dependent on their previous engagement.

We are now entering the usability-testing phase of the project. The issues we expect to face include the following:

I some phones need to swap a code with the server (handshake problems),

I some people don't enable Bluetooth,

I some people don't understand Bluetooth, and

I some phones have memory limitations.

The infrastructure independence of the server is limited by the need to make data backups as a protection against data corruption.

There are additional issues related to allowing people to send their media to the server. The extent to which viruses will present an operational issue remains to be seen. Furthermore, automatically recirculating user-generated media back to the public is culturally impossible in the university context. A system for moderating the content will have to be established, and it's not yet clear to what extent that system can be programmatic.

\section{Locative stories}

According to Ronald Lenz, future engagement with the culturally convergent Internet might be automatically context sensitive. ${ }^{2} \mathrm{We}$ could describe this paradigm as Mobile 2.0 (context-sensitive, mobile-based social software meets participatory media). We hope to see the traditional one-way production, distribution, and consumption cycle become more and more multidirectional and iterative. Existing applications such as Cityware for Facebook (see http://www.cityware.org.uk and http://apps.facebook.com/cityware), developed

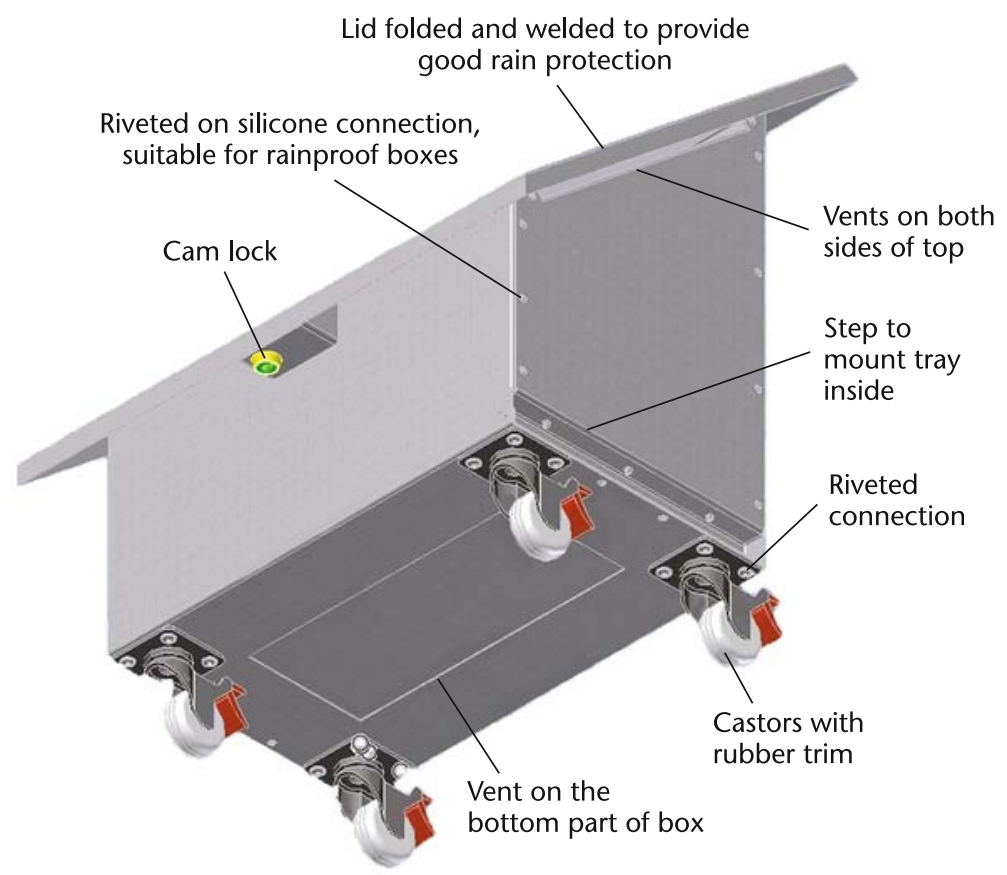

by computer scientists at the University of Bath, use Bluetooth IDs to gather statistics on your proximity to your Facebook friends. We envision extending such systems to selectively send media between a group of friends when they are within the Bluetooth server's range.

According to Roberts et al., "[t]he challenge for the human-centred city is to reconcile the personal and the local with the metropolitan." ${ }^{3}$ We're interested in urban environments and the human issues that they entail—urban planning, architecture, sustainability, pollution, traffic flows, and so on. These themes suggest some of the stories we can derive from urban locations.

We want to design subtle urban spaces, layered with responsive, personal, and even private media, telling local, on-the-fly narratives and poems that are invented through peer-to-peer interaction. However, such storytelling must resolve various aesthetic challenges, which are ultimately derived from the size of mobile devices.

Psychogeography is a term coined by Guy Debord to describe "the study of the precise laws and specific effects of the geographical environment, consciously organized or not, on the emotions and behaviour of individuals." Ivan Chtcheglov ${ }^{4}$ proposed a psychogeographic ${ }^{5}$ poetics, in which cityscapes become laden with virtual identities such as the bizarre quarter or the sinister quarter: the very names
Figure 4. Technical drawing of the solarpowered Bluetooth server. Wings affixed at the angled lid will increase the total area available for the solar panels. 
elicit locative stories yet to be told. The mobile phone is the perfect device for the delivery and sharing of these types of mediated experiences.

MM

\section{Acknowledgment}

This article is a version of a paper originally presented at the Screenscapes conference, Sydney University, 2007. Thanks to Ge PeiQi for technical information about the Bluetooth server's software.

\section{References}

1. P. Golding, "Air tagging...," blog, 26 March 2005, http://wirelesswonders.blogspot.com/2005/03/ air-tagging.html.

2. R. Lenz, Locative Media, 2005; http://spresearch. waag.org/images/LocativeMedia.pdf.
3. M. Roberts et al., "Place and Space in the Networked City: Conceptualizing the Integrated Metropolis." J. Urban Design, vol. 4, no. 1, 1999, pp. 51-66.

4. I. Chtcheglov, Formulary for a New Urbanism, 1953; http://library.nothingness.org/articles/SI/en/ display/1.

5. G.-E. Debord, "Introduction to a Critique of Urban Geography," Les Lèvres Nues [New Pages], no. 6, 1955; http://library.nothingness.org/articles/all/ en/display/2.

Contact author Jenny Weight at jenny.weight@rmit. edu.au.

Contact editor Norie Neumark at n.neumark@uts. edu.au.

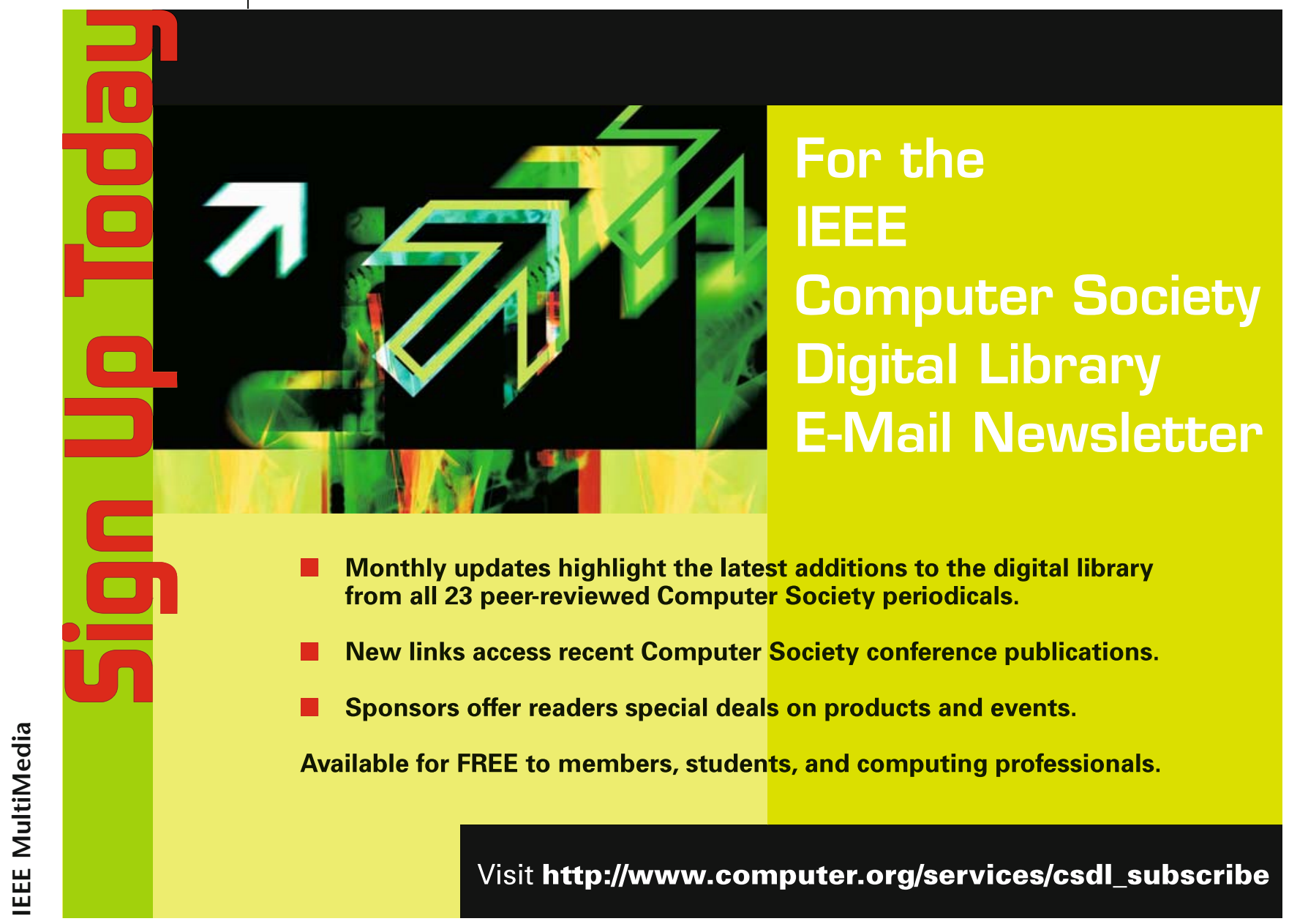

